\title{
Mammalian Target of Rapamycin (mTOR) Regulates Cellular Proliferation and Tumor Growth in Urothelial Carcinoma
}

\author{
Donna E. Hansel, ${ }^{\star \dagger} \S \S$ Eric Platt, ${ }^{*}$ \\ Mohammed Orloff, ${ }^{\dagger}$ Jyoti Harwalker, ${ }^{*}$ \\ Swathi Sethu, ${ }^{*}$ Jessica L. Hicks, ${ }^{\pi}$ \\ Angelo De Marzo, "Roxanne E. Steinle, * \\ Eric D. Hsi, ${ }^{*}$ Dan Theodorescu, \\ Christina B. Ching, ${ }^{\S}$ and Charis Eng ${ }^{\dagger \star * *}$ \\ From the Department of Anatomic Pathology, the Genomic \\ Medicine Institute, ${ }^{\dagger}$ the Taussig Cancer Institute, ${ }^{\ddagger}$ and the \\ Glickman Urological and Kidney Institute, ${ }^{\circledR}$ The Cleveland Clinic, \\ Cleveland, Obio; the Department of Pathology, "The Johns \\ Hopkins Hospital, Baltimore, Maryland; the Department of \\ Urology, University of Virginia, Charlottesville, Virginia; and the \\ Department of Genetics and CASE Comprehensive Cancer \\ Center,** Case Western Reserve University School of Medicine, \\ Cleveland, Ohio
}

Mammalian target of rapamycin (mTOR) signaling has been associated with aggressive tumor growth in many cancer models, although its role in urothelial carcinoma (UCC) has not been extensively explored. Expression of phosphorylated mTOR (P-mTOR) and a downstream target, ribosomal $\mathbf{S 6}$ protein (P-S6), was identified in $74 \%(90 / 121)$ and $55 \%(66 / 121)$ of muscle-invasive UCCs, respectively. P-mTOR intensity and $\%$ positive cells were associated with reduced diseasespecific survival $(P=0.04, P=0.08$, respectively). Moreover, P-mTOR intensity corresponded to increased pathological stage $(P<0.01)$, and mTOR activity was associated with cell migration in vitro. In addition, mTOR inhibition via rapamycin administration reduced cell proliferation in UCC cell lines RT4, T24, J82, and UMUC3 in a dose-dependent manner to $6 \%$ of control levels and was significant at $1 \mathrm{nmol} / \mathrm{L}$ in J82, T24, and RT4 cells $(P<0.01, P<0.01, P=0.03$, respectively) and at $10 \mathrm{nmol} / \mathrm{L}$ in UMUC3 cells $(P=$ 0.03). Reduced proliferation corresponded with reduced P-S6 levels by Western blot, and effects were ablated by pretreatment of cells with mTOR-specific siRNA. No effects of rapamycin on apoptosis were identified by TUNEL labeling or PARP cleavage. Administration of rapamycin to T24-xenografted mice resulted in a 55\% reduction in tumor volume $(P=$ $0.03)$ and a $40 \%$ reduction in proliferation $(P<0.01)$ compared with vehicle-injected mice. These findings indicate that mTOR pathway activation frequently occurs in UCC and that mTOR inhibition may be a potential means to reduce UCC growth. (Am J Pathol 2010, 176:3062-3072; DOI: 10.2353/ajpath.2010.090872)

Bladder cancer occurs in multiple forms, the most common of which is urothelial carcinoma (UCC), which represents $>90 \%$ of all bladder cancers. ${ }^{1}$ Approximately 30 to $50 \%$ of patients with invasive bladder cancer into the muscular wall of the bladder will develop metastatic disease and die within 2 years of diagnosis. ${ }^{2}$ In addition, virtually all patients diagnosed with distant UCC metastases will succumb to disease. ${ }^{3}$ Currently, the standard treatment modality for muscle-invasive bladder cancer is radical cystectomy; systemic chemotherapy is generally reserved for patients with metastatic disease, although these treatment regimens provide only a limited longterm benefit with only rare reports of complete remission. ${ }^{4,5}$ In light of these clinical outcomes, identification of new therapeutic targets is needed to define potential additional treatment avenues for these patients.

Activation of the mammalian target of rapamycin (mTOR) signaling pathway occurs in many cancers and has recently been shown to correlate with more aggressive disease behavior, ${ }^{6-9}$ although it has not been examined in great detail

Supported in part by a pilot program grant from the Cleveland Clinical and Translational Sciences Collaborative (CTSC) of Case Western Reserve University (to D.E.H.), the American Cancer Society Ohio Pilot Research grant (to D.E.H.), a KL2 Career Development Award (to D.E.H.), and the M. Frank and Margaret Domiter Rudy Chair fund. C.E. is a recipient of the Doris Duke Distinguished Clinical Scientist Award, is an ACS Clinical Research Professor, and is the Sondra J. and Stephen R. Hardis Chair of Cancer Genomic Medicine at the Cleveland Clinic.

Accepted for publication February 25, 2010

Supplemental material for this article can be found on http://ajp. amjpathol.org.

Address reprint requests to Donna E. Hansel, M.D., Ph.D., 9500 Euclid Avenue, Desk L25, Cleveland, OH 44195. E-mail: hanseld@ccf.org. 
in UCC. Activation of mTOR occurs via a multistep process that includes upstream phosphoinositide-3 kinase (PI3K) and AKT activation, leading to phosphorylation and inactivation of the tuberous sclerosis complex 1 and 2 (TSC1/ TSC2) heterodimer. ${ }^{10,11}$ Inactivation of this heterodimer results in release of Rheb inhibition and subsequent mTOR activation by means of Rheb GTPase activity. Once activated, mTOR can induce increased mRNA translation or regulate the actin cytoskeleton via differential association Rictor and Raptor proteins. ${ }^{10,11}$ Ultimately, mTOR activity regulates the effects of a number of downstream molecules important in cellular growth, including p70 S6 kinase-1 (S6K) and elongation-initiation factor $4 \mathrm{E}$ binding protein-1 (4E-BP1). Selective inhibition of the mTOR pathway can be achieved using rapamycin or rapamycin analogs temsirolimus (CCl-779, Wyeth Pharmaceuticals) and everolimus (RAD001, Novartis), which are currently in use in numerous clinical trials for solid tumors, with promising results in patients with advanced renal cell carcinoma. ${ }^{12,13}$

To further investigate the potential role of mTOR signaling and inhibition in UCC of the bladder, we used human cancer specimens, xenograft models, and in vitro analysis to determine the effects of mTOR on cellular proliferation, apoptosis, tumor growth, and clinical outcomes in this cancer population.

\section{Materials and Methods}

\section{Patient Specimens}

Permission for this study was obtained from The Cleveland Clinic Institutional Review Board. Specimens included archived paraffin blocks from patients who underwent radical cystectomy or cystoprostatectomy for muscle-invasive UCC (pathological stage pT2 or greater) between the years 1998 to 2007. All specimens were received in the surgical pathology suite on ice within 10 minutes postcystectomy, and tissue was immediately placed into 10\% buffered formalin for routine processing. Primary bladder tumors that were either nonmetastatic $(n=52)$ or metastatic to regional lymph nodes $(n=69)$ were used for analysis. Paired lymph node metastases from the latter group were available in 59 cases for analysis. Patient demographics, clinicopathologic features, and outcomes are presented in Table 1. In addition, noninvasive low- $(n=20)$ and high-grade $(n=20)$ papillary UCCs identified on biopsy were used for comparison for P-mTOR and P-S6 expression.

\section{Immunohistochemistry}

Tissue microarrays were prepared from both nonmetastatic and metastatic UCCs. Each specimen was represented by four 1.0-mm cores on tissue microarray to obtain adequate representation of different regions of neoplastic cells to assess for intratumoral heterogeneity and which has been demonstrated to adequately represent tissue variation within UCC. ${ }^{14}$ Slides were deparaffinized in fresh xylenes and rehydrated through sequential-graded ethanol steps. For P-S6, antigen retrieval was performed by citrate buffer incubation [ $18 \mathrm{mmol} / \mathrm{L}$ sodium citrate ( $\mathrm{pH}$ 6.0)] using a household vegetable steamer for 60 minutes. Slides were incubated for 5 minutes with 3\% hydrogen peroxide, washed in TBS/T [20 mmol/L Tris, 140 $\mathrm{mmol} / \mathrm{L} \mathrm{NaCl}, 0.1 \%$ Tween 20 (pH 7.6)], and incubated in appropriate antibody dilution for P-S6 (Thr389, 1:50, Cell Signaling Technologies) overnight at $4^{\circ} \mathrm{C}$. P-S6 immunostaining was validated by a secondary analysis with Ser240/ 244 antibody (1:50, Cell Signaling Technologies) with no significant differences in pathway activation identified. Normal saline was substituted for the primary antibody in control sections. The avidin-biotin-peroxidase complex method from DAKO (Glostrup, Denmark) was used, and slides were subsequently counterstained with hematoxylin. P-mTOR immunohistochemistry was performed using an automated stainer (Ventana Medical Systems, Tucson, AZ) with monoclonal antibody against P-mTOR (clone 49F9; Cell Signaling Technologies). Assessment of immunohistochemical labeling of the tissue microarrays was performed by one of the authors (D.E.H.). Immunostaining was scored semiquantitatively including both intensity of stain ( 0 , no staining; 1 , weak staining; 2 , moderate staining; 3 , intense staining) and percentage of immunoreactive cells within the lesion. Comparison of immunolabeling between populations was performed using the Fisher exact test.

Table 1. Patient Demographics and Clinical Outcomes

\begin{tabular}{|c|c|c|c|}
\hline & Total primary carcinomas & Nonmetastatic subset & Metastatic subset \\
\hline Patient number, $\mathrm{n}$ & 121 & 52 & 69 \\
\hline \multicolumn{4}{|l|}{ Patient age, y } \\
\hline Mean & 67 & 68 & 66 \\
\hline Median & 68 & 71 & 65 \\
\hline Range & $33-89$ & 33-89 & $43-84$ \\
\hline Male:female ratio & $98: 23$ & $45: 7$ & $53: 16$ \\
\hline \multicolumn{4}{|l|}{ Pathologic stage, pT } \\
\hline pT1 & $3(2 \%)$ & $2(4 \%)$ & $1(2 \%)$ \\
\hline pT2 & $29(24 \%)$ & $16(31 \%)$ & $13(19 \%)$ \\
\hline pT3 & $84(69 \%)$ & $34(65 \%)$ & $50(72 \%)$ \\
\hline pT4 & $5(4 \%)$ & $0(0 \%)$ & $5(7 \%)$ \\
\hline Positive margin & $32(27 \%)$ & $12(23 \%)$ & $20(29 \%)$ \\
\hline Clinical follow-up available & $118(98 \%)$ & $50(96 \%)$ & $68(99 \%)$ \\
\hline Mean follow-up, mo & 28 & 33 & 24 \\
\hline Median follow-up, mo & 22 & 27 & 17 \\
\hline Subsequent metastases & 38 (32\%) & $15(30 \%)$ & $23(33 \%)$ \\
\hline Dead of disease & $74(63 \%)$ & $25(50 \%)$ & $49(71 \%)$ \\
\hline
\end{tabular}




\section{Statistical Analysis of P-mTOR and P-S6 to Clinical Parameters}

Statistical analysis was performed using SPSS version 17.0 (SPSS Inc., Chicago, IL) and Statistica (Statsoft Inc., Tulsa, OK). All tests were two-tailed, analyzed at a significance level of $P \leq 0.05$, with a statistical trend defined as $P \leq 0.1$. The Mann-Whitney test was used to compare the probability distributions from two independent groups, one-way analysis of variance was used to compare means between independent groups. Pearson correlation was used to determine the linear dependence between two variables. Regression (Logistic, Ordinal) was used for predicting probability for data, and survival analysis was performed using the Cox Proportional Hazards model. The end point in survival analysis was diseaserelated death, measured in time as a function of months from surgery until death. The groups mTOR and S6 intensity were both dichotomized along their median (0-1) into 0 , and (2-3) into 2.

\section{Cell Culture and Cell Culture Assays}

UCC cell lines T24, RT4, UMUC3, and J82 were purchased from the American Type Culture Collection cell line bank (ATCC, Manassas, VA). Cells were grown on 60-mm Falcon dishes (Becton Dickinson Labware, Franklin Lakes, NJ) in RPMI-1640 (GIBCO, Invitrogen Corp., Grand Island, NY) supplemented with 10\% fetal bovine serum (GIBCO) and, for labeling analysis, subsequently plated onto LAB-TEK II 4-well chambered slides (Fisher Scientific, Pittsburgh, PA). Cells were maintained at $50 \%$ confluence. Twenty-four hours before rapamycin addition (Santa Cruz Biotechnology, Inc, Santa Cruz, CA), the cell culture medium was replaced with RPMI/0.5\% fetal bovine serum. After serum starvation, graded doses of rapamycin (1 picomolar to 1 micromolar concentration for 48 hours) were applied to cells. For proliferation assays, cells were subsequently labeled for 6 hours using the BrdU Labeling Kit I (Roche Diagnostics, Indianapolis, IN) and stained per protocol. Experiments were performed in triplicate, and cell counts per treatment condition included, on average, 1291 (J82), 1015 (T24), 1738 (RT4), and 588 (UMUC3) cells. For apoptosis analysis, cells were analyzed using the in Situ Cell Death Detection Kit (Roche Applied Science, Indianapolis, IN) following the same treatment protocol described for proliferation assays. Cell migration assays were performed using the Boyden Chamber motility assay (Costar transwell permeable support, $8 \mu \mathrm{mol} / \mathrm{L})$ using 60,000 cells per well. Cells were treated with DMSO or varying concentrations of rapamycin in DMSO for 36 hours, followed by fixation with absolute methanol for 10 minutes and visualization by crystal violent stain (Mallinckrodt Baker, Phillipsburg, $\mathrm{NJ}$ ). Comparison between treatment populations was performed by two-tailed Student $t$ test analysis. For immunohistochemical analysis, cells were trypsinized, resuspended in RPMI-1640, and spun to collect the cell pellet, which was fixed with $10 \%$ cold neutral buffered saline overnight at $4^{\circ} \mathrm{C}$. The following day, cells were centrifuged, washed twice in PBS, and incubated with plasma and thrombin to form a cell clot, which was embedded in paraffin using a standard tissue-processing cassette and processor, and $5 \mu \mathrm{mol} / \mathrm{L}$ sections were obtained on charged slides (Cardinal Health, Dublin, $\mathrm{OH})$. Immunohistochemical analysis was performed as described for paraffin-embedded tissue specimens. Scrambled control and mTOR siRNA were obtained from Cell Signaling Technologies and cells were transfected with OPTI-MEMI (Gibco).

\section{Cell Extracts and Western Blotting}

Confluent cells were extracted into lysis buffer consisting of $25 \mathrm{mmol} / \mathrm{L}$ Tris- $\mathrm{HCl}$ ( $\mathrm{pH} 7.6), 150 \mathrm{mmol} / \mathrm{L} \mathrm{NaCl}, 1 \%$ Triton-X-100, 1 EDTA, 0.1\% SDS, protease inhibitor cocktail (Sigma, St. Louis, MO), and phosphatase inhibitor cocktail (Roche Diagnostics, Indianapolis, IN), centrifuged at $10,000 \mathrm{~g}$ for 30 minutes at $4^{\circ} \mathrm{C}$, and the supernatant containing soluble proteins was collected. Protein concentration was measured using the Bio-Rad protein assay reagent (Bio-Rad Laboratories, Hercules, CA). Twenty-five $\mu \mathrm{g}$ of total protein per sample was fractionated on a standard SDS-polyacrylamide gel containing $10 \%$ acrylamide/0.8\% bis-acrylamide and transferred via semidry transfer cell (Trans-blot SD, Bio-Rad) to nitrocellulose membranes (Millipore, Bedford, MA) in $48 \mathrm{mmol} / \mathrm{L}$ Tris, $39 \mathrm{mmol} / \mathrm{L}$ glycine, $0.0375 \%$ SDS, and $20 \%$ methanol. Prestained molecular weight standards were run concurrently with protein samples (Lonza, Rockland, $\mathrm{ME})$. Blots were blocked with $1 \%$ bovine serum albumin diluted in $10 \mathrm{mmol} / \mathrm{L}$ Tris- $\mathrm{HCl}(\mathrm{pH} \mathrm{7.5)}$ and $150 \mathrm{mmol} / \mathrm{L}$ $\mathrm{NaCl}$ containing $0.05 \%$ Tween 20 (TBS/T; phosphorylated p70S6kinase-1 antibody) or in 5\% Carnation instant milk in TBS/T (for remaining antibodies) for 1 hour at room temperature. Antibodies included P-mTOR (1:1000), total mTOR (1:1000), P-p70S6kinase-1 (P-S6K; 1:1000), total S6K (1:1000), P-S6 (1:1000), total S6 (1:1000), P-AKT (Ser473; 1:500), P-AKT (Thr308; 1:500); total AKT (1: 1000), Rictor (1:1000), Raptor (1:1000), TSC1 (1:1000), TSC2 (1:1000), and PARP (1:2000), all from Cell Signaling Technologies. Antibodies for PTEN (clone 6H2.1) were obtained from Cascade Bioscience (Winchester, MA) and used at a dilution of 1:500. Blots were incubated with antibody overnight at $4^{\circ} \mathrm{C}$ in blocking solution and rinsed, followed by incubation for 2 hours at room temperature with alkaline phosphatase conjugated anti-rabbit IgG antibody (1:30,000; Amersham Corp.) and visualized using the Enhanced Chemiluminescence Kit (Amersham Corp.). Proteins were visualized using chemifluorescence scanning Storm 860 Molecular Imager (GMI, Ramsey, MN). After primary antibody incubation, blots were stripped in $62.5 \mathrm{mmol} / \mathrm{L}$ Tris, $\mathrm{pH} 6.8$, containing $100 \mathrm{mmol} / \mathrm{L}[\beta]$-mercaptoethanol and 2\% SDS for 30 minutes at $50^{\circ} \mathrm{C}$. Western blots were reprobed using a 1:1000 dilution of either total mTOR or total S6K (Cell Signaling Technologies) and reprobed with a 1:1000 dilution of rabbit polyclonal anti-actin antibody (Sigma Chemical Company, St. Louis, MO) for 1 hour. 


\section{PTEN Gene Analysis}

Genomic DNA was extracted from T24 and UMUC3 cells and the PTEN gene was analyzed via standard PCR, using GC-clamped intronic primers that encompass each of the 9 PTEN exons and flanking intronic sequences, followed by denaturing gradient gel electrophoresis analysis, ${ }^{15}$ which has proven more than 99\% sensitive and specific for intragenic "small" mutations. Identified denaturing gradient gel electrophoresis variants were next subjected to DNA sequencing ${ }^{16}$ to determine specific sequence variations within targeted regions.

\section{Mouse Xenograft Studies}

Xenograft studies were performed as a collaborative effort with University of Virginia under institutional IACUC approval. Subcutaneous inoculation of 6-week-old nude mice was performed using $10^{6}$ T24 cells in 50\% matrigel (BD Biosciences, San Jose, CA) for both control $(n=9)$ and rapamycin $(n=8)$ groups. Mice were examined and weighed weekly until the establishment of visible tumors.
Once established, mice were given twice-weekly intraperitoneal injections of either $2.5 \mathrm{mg} / \mathrm{kg}$ rapamycin in DMSO or vehicle alone. ${ }^{17}$ Subcutaneous tumors were measured twice weekly for 4 weeks using calipers, and tumor volume was calculated in $\mathrm{mm}^{3}$, with statistical significance calculated via the Wilcoxon Signed Ranks Test Tumors were embedded in formalin, sectioned, and subsequently immunostained with Ki-67 (predilute; Ventana Medical Systems, Inc., Tucson, AZ) using the Ventana Benchmark XT. Statistical analysis of proliferation indices between populations was performed using the Student $t$ test.

\section{Results}

\section{Activation of the mTOR Pathway is Common in UCC}

To examine the potential role of the mTOR pathway in UCC, we first examined the frequency of mTOR activation in a consecutive cohort of 121 muscle-invasive UCCs using well-characterized antibodies targeting phosphor-
$\mathrm{H} \& \mathrm{E}$
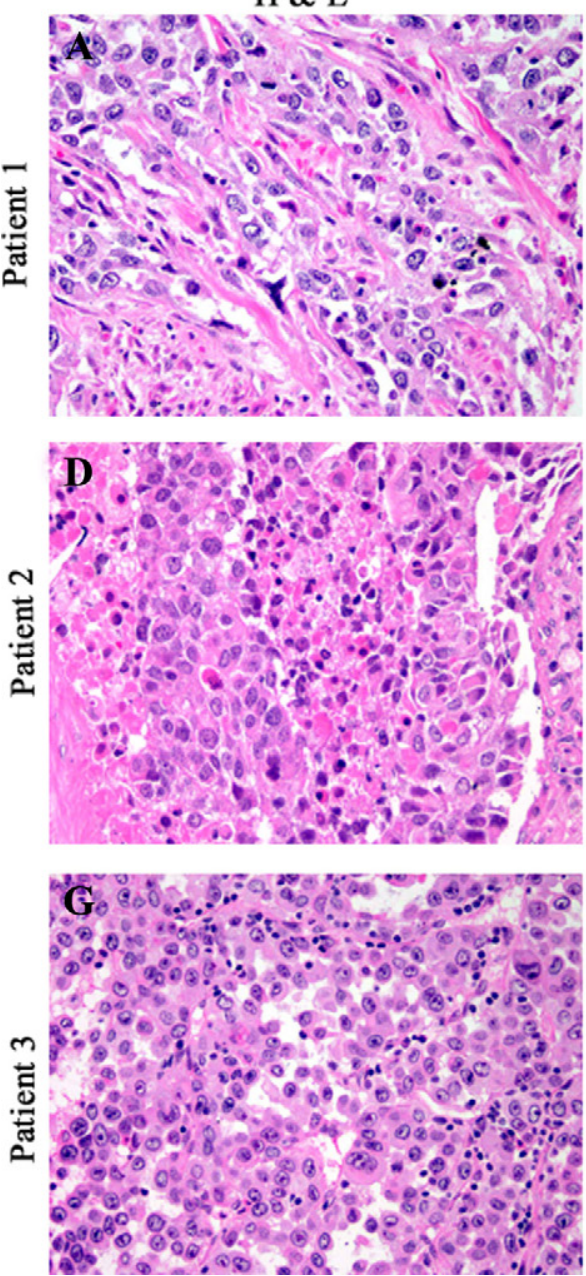

P-mTOR
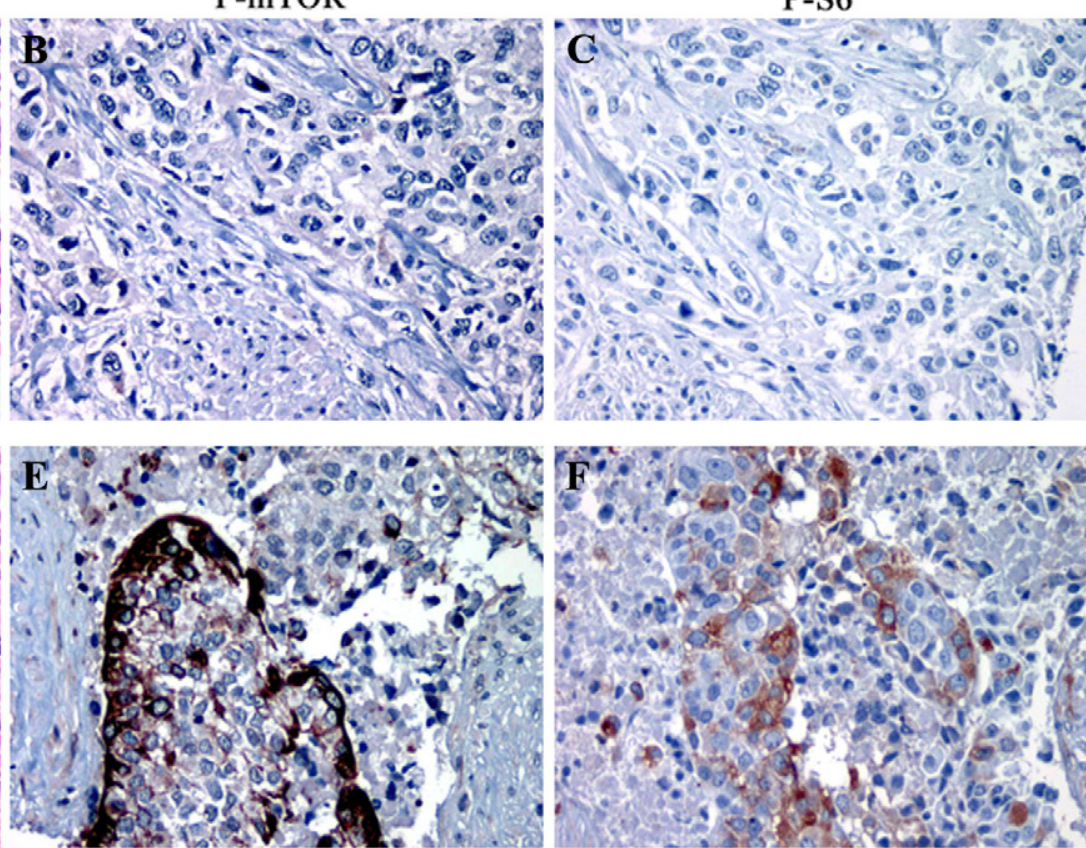
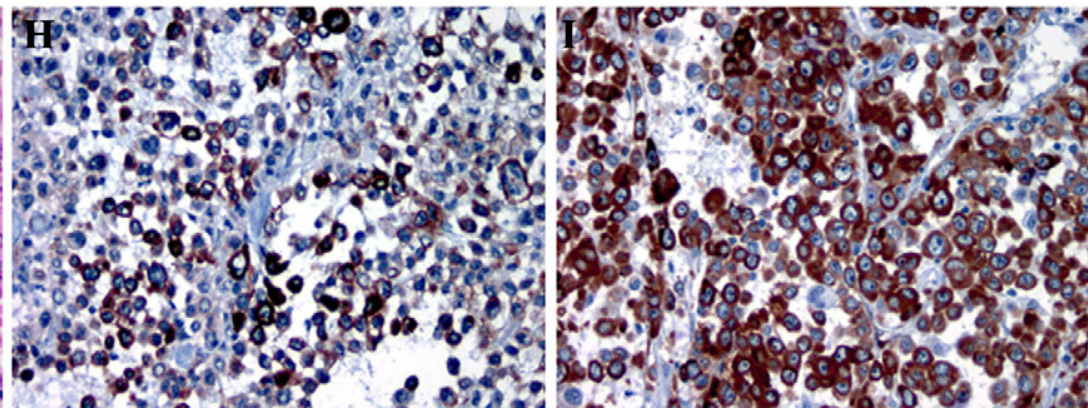

Figure 1. Immunohistochemical analysis of human UCC specimens demonstrates a range of P-mTOR and P-S6 expression, including $1+$ reactivity in $<5 \%$ of cell (A-C), 2 to $3+$ immunoreactivity in $25 \%$ of cells (D-F), and 2 to $3+$ immunoreactivity in $100 \%$ of tumor cells (G-I). Concordant immunohistochemical intensity and percent positive cells was present in $60 \%$ of metastatic and $71 \%$ of nonmetastatic primary carcinomas. 
A



C



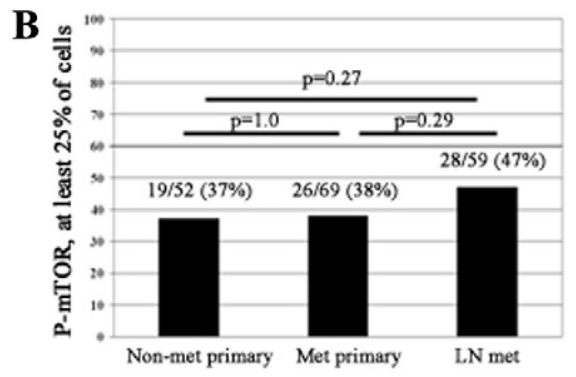

D

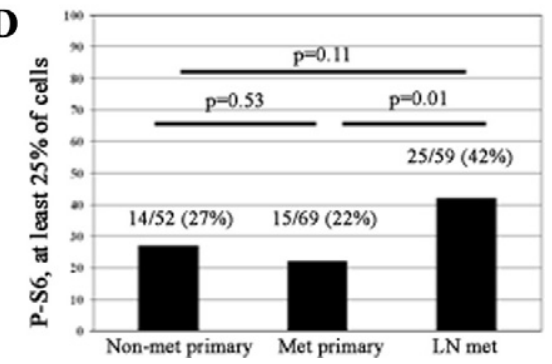

Figure 2. Comparison of P-mTOR and P-S6 in tensity and percent immunoreactive cells in primary nonmetastatic UCC (Non-met primary), primary UCC associated with metastases (Met primary), and paired lymph node metastases (LN met). A: 2 to $3+$ P-mTOR immunoreactivity is present in the majority of UCC cases. B: Approximately one-third of all UCC cases demonstrate PmTOR in at least $25 \%$ of tumor cells. C: 2 to $3+$ P-S6 immunoreactivity is present in a lower percentage of UCC cases and shows a significant increase in lymph node metastases $(P<0.01$, Fisher exact test); D, Similar to P-mTOR, of 2 to $3+$ a large proportion of P-S6 expressing UCCs demonstrate expression in at least $25 \%$ of tumor cells, with a significant increase in expression in lymph node metastases ( $P=0.01$, Fisher exact test) ylated mTOR (P-mTOR, Ser2448), a marker of mTOR activation, ${ }^{18,19}$ and phosphorylated S6 (P-S6), a downstream target of mTOR. Specimens included 52 cases of primary UCCs without metastases ("non-metastatic UCCs") and 69 cases of primary UCCs ("metastatic UCCs") with concurrent regional lymph node metastases (Table 1). Of these latter cases, 59 paired lymph node metastases were available for analysis. No significant differences in patient age, gender, pathological stage, margin status, or clinical follow-up were present between populations. Semiquantitative analysis of tumor cells expressing either P-mTOR or P-S6 was performed and was tallied as a continuous score from 0 to $100 \%$ and as a range of immunoreactivity (0 to 3+ intensity; Figure 1, A-I).

Phosphorylation of mTOR was identified in 90/121 $(74 \%)$ primary UCCs, with the majority of tumors demonstrating 2 to $3+$ immunoreactivity (82/121; 68\%; Figure 2A). P-mTOR expression was present in at least $25 \%$ of cells in approximately half of all P-mTOR-expressing UCCs (19/52 non-metastatic and 26/69 metastatic UCCs; Figure 2B), with the remainder demonstrating more focal P-mTOR expression. No significant difference was apparent either between nonmetastatic or metastatic primary UCC or between primary UCCs and lymph node metastases when comparing either intensity of expression $(P=0.56$ and $P=0.85$, respectively) or percentage of positive cells ( $P=1.0$ and $P=0.29$, respectively).

Although phosphorylation of mTOR has been used extensively as a biomarker to indicate activation of the mTOR pathway in cancer cells, we additionally examined the phosphorylation of ribosomal S6 protein, a downstream target of mTOR. In contrast to P-mTOR levels, P-S6 demonstrated a slight reduction in both intensity of expression and percentage of positive cells, with the greatest difference identified in the primary UCC populations. Overall, expression of P-S6 was identified in 66/121 (55\%) of all primary UCCs examined. P-S6 intensity of 2 to $3+$ was reduced to $52 \%(27 / 52)$ of nonmetastatic and $35 \%$ of metastatic primary UCCs (Figure 2C). Similarly, fewer cases demonstrated at least $25 \%$ tumor cell immunoreactivity in the pri- mary UCC component, which was present in only $27 \%$ $(14 / 52)$ of nonmetastatic and $22 \%(15 / 69)$ of metastatic lesions (Figure 2D). Robust expression of P-S6, however, remained constant in lymph node metastases, with a significant increase in P-S6 status, defined as both intensity of staining and percentage of positive cells, in the lymph node metastases versus the paired primary carcinoma $(P<$ 0.001 and $P=0.01$, respectively).

Concordance between P-mTOR and P-S6 status, defined as identical intensity and \%positive cells within $20 \%$, was present in 31/52 non-metastatic (60\%) and 49/69 (71\%) of metastatic primary carcinomas, with the majority of discordant cases representing a reduction in P-S6 levels relative to P-mTOR, as demonstrated in Figure 2.

We next evaluated a set of noninvasive lesions, including 20 low-grade papillary UCCs and 20 high-grade papillary UCCs. In low-grade lesions, P-mTOR intensity of 2 to 3 was present in 13/20 (65\%) lesions and was present in approximately $50 \%$ of cells on average (range, 5 to $80 \%$ ). Similar to that identified in muscle-invasive UCCs, P-S6 expression was more limited, present in $36 \%$ of cells with an average intensity of 2 . Of note, P-S6 expression in noninvasive low-grade papillary UCC appeared to be restricted in many cases to the upper half of the urothelium (Figure 3A). Similar results were identified for noninvasive high-grade papillary UCC (P-mTOR mean \%cells $31 \%$, range 5 to $80 \%$, mean intensity 2; P-S6 mean \%cells $38 \%$, range 10 to $100 \%$, mean intensity 2$)$. In contrast to low-grade lesions, high-grade papillary UCC often demonstrated full-thickness expression of P-S6 (Figure 3B).

\section{P-mTOR Status Predicts Worsened Pathological Stage and Reduced Survival}

As phosphorylation of mTOR and S6 appeared to be increased in lymph node metastases, an indicator of aggressive disease, we next examined the relationship between P-mTOR and P-S6 status and clinicopathologic parameters, including positive margin status, pathologi- 
A



Figure 3. P-S6 immunolabeling in noninvasive papillary UCC demonstrates immunoreactivity in a significant proportion of low- and high-grade lesions. A: Low-grade lesions frequently demonstrated limited immunoreactivity to the upper half of the urothelium; B: In contrast, high-grade lesions often showed full-thickness staining. cal stage (depth of invasion), development of subsequent metastases, and disease-specific survival.

P-mTOR intensity, defined and dichotomized as 0 to $1+$ (no to weak staining) or 2 to $3+$ (moderate to strong staining), was evaluated against pathological parameters using the Mann-Whitney test. The probability distributions were found to differ for depth of invasion $(P<$ $0.001)$, and ordinal regression analysis was performed. Increased P-mTOR intensity was found to be significantly associated across all categorical levels (0 to 2$)$ ) of invasion depth relative to the highest level of $3(P<0.01, P=$ $0.05, P=0.02$, respectively), with a stronger $P-m T O R$ intensity yielding a more aggressive invasion. P-mTOR intensity also demonstrated a significant inverse relationship with length of survival using one-way analysis of variance analysis $(P=0.04)$, which is graphically represented (Figure 4A). In addition, increased percentage of P-mTOR-positive cells was also independently associated with increased depth of invasion on a continuous regression $(P<0.01)$ and showed a trend toward negative correlation with length of disease-specific survival using Cox Proportional Hazards model $(P=0.08$, Figure $4 B$ ). No significant association between either P-mTOR intensity or percentage of P-mTOR positive cells was identified for margin status at cystectomy or the presence of subsequent metastatic disease.

A similar analysis was performed for P-S6 in primary UCCs of the bladder. P-S6 intensity was found to be positively associated with increased depth of invasion across most ordinal levels relative to $3(P<0.01, P<$ $0.01, P=0.77$, respectively) No significant association was identified between percentage of P-S6-positive cells and increased depth of invasion $(P=0.47)$.

As mTOR activity appeared to correlate with pathological stage, we assessed the effects of mTOR activity on UCC migration in vitro. Using the Boyden Chamber assay, we plated T24 and UMUC3 cells into control (DMSO-solvent containing) or rapamycin-containing (10 nmol/L, $1 \mu \mathrm{mol} / \mathrm{L})$ media for 36 hours. Both T24 and UMUC3 cells treated with rapamycin, a general $\mathrm{mTOR}$ inhibitor, demonstrated a reduction in cells that were successfully able to migrate through the insert. Under control conditions, 285 cells ( \pm 35 ) T24 cells and 935 UMUC3 cells ( \pm 40 ) migrated through the insert. Cell migration was reduced in T24 and UMUC3 cells, respectively, to $39 \%$ and $70 \%$ with $10 \mathrm{nmol} / \mathrm{L}$ rapamycin and $35 \%$ and $45 \%$ with $1 \mu \mathrm{mol} / \mathrm{L}$ rapamycin. These findings suggest that mTOR activity may play a role in potentially mediating cell migration in UCC cells.

\section{Inhibition of mTOR Reduces UCC Cell Proliferation and Is Associated with Reduced Downstream Target Phosphorylation}

Because of the frequent nature of mTOR pathway activation in UCC, we next sought to determine the role of mTOR inhibition in mediating cell proliferation in vitro. We used the immortalized UCC cell lines T24, RT4, J82, and UMUC3, which demonstrated baseline phosphorylation of mTOR and S6 by Western blot analysis. Cells were
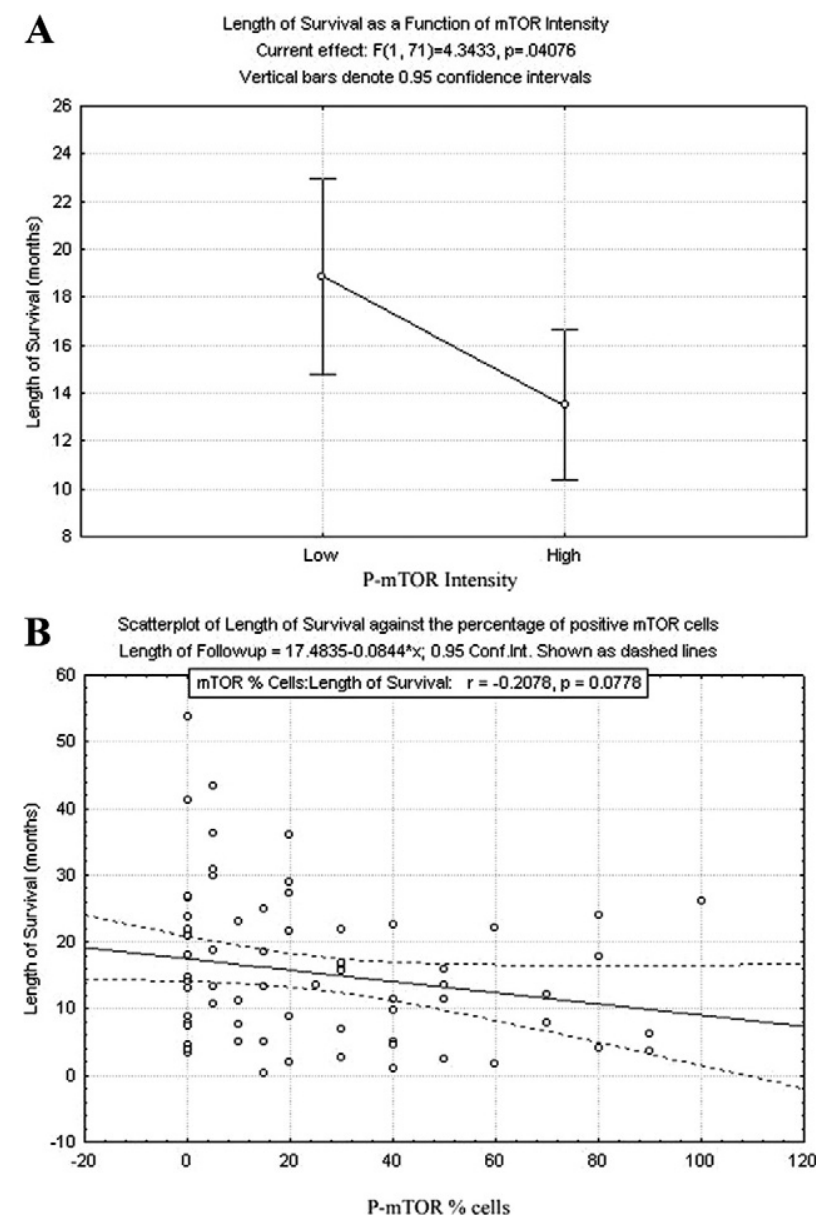

Figure 4. Cox Proportional Hazards model analysis was performed for $\mathbf{A}$ P-mTOR intensity ( 0 to $1+$ versus 2 to $3+; \exp (\beta)=1.693, P=0.041)$. B: Percentage of cells immunoreactive for P-mTOR as quartiles $\exp (\beta)=1.007$, $P=0.078$ ). Both P-mTOR intensity and percentage of positive cells were associated with reduced disease-specific survival. 
A

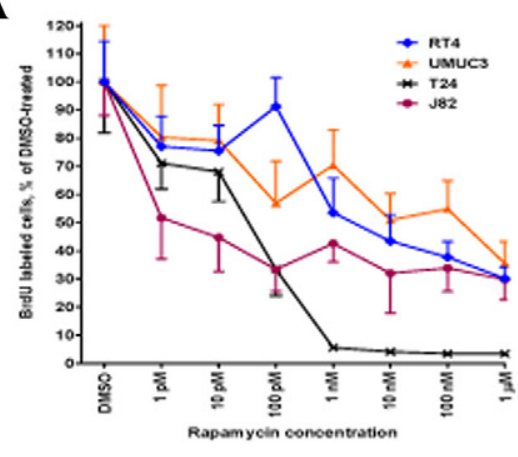

Figure 5. A: Incubation of J82, T24, RT4, and UMUC3 UCC cell lines with increasing doses of rapamycin demonstrated a dose-dependent reduction in BrdU-labeled cells relative to DMSO-treated control cells. All experiments were performed in triplicate. B-G, Robustness of response to rapamycin administration parallels the intensity of P-mTOR $(\mathbf{C}, \mathbf{F})$ and P-S6 $(\mathbf{D}, \mathbf{G})$ expression in both T24 and UMUC3 cells Western blot analysis of T24 cells (H) and UMUC3 cells (I) after treatment with DMSO (Ctrl) or rapamycin $(1 \mathrm{pM}$ to $1 \mu \mathrm{mol} / \mathrm{L})$ shows a reduction in P-S6K and P-S6 levels at low-dose rapamycin that parallels reduction in cell proliferation.

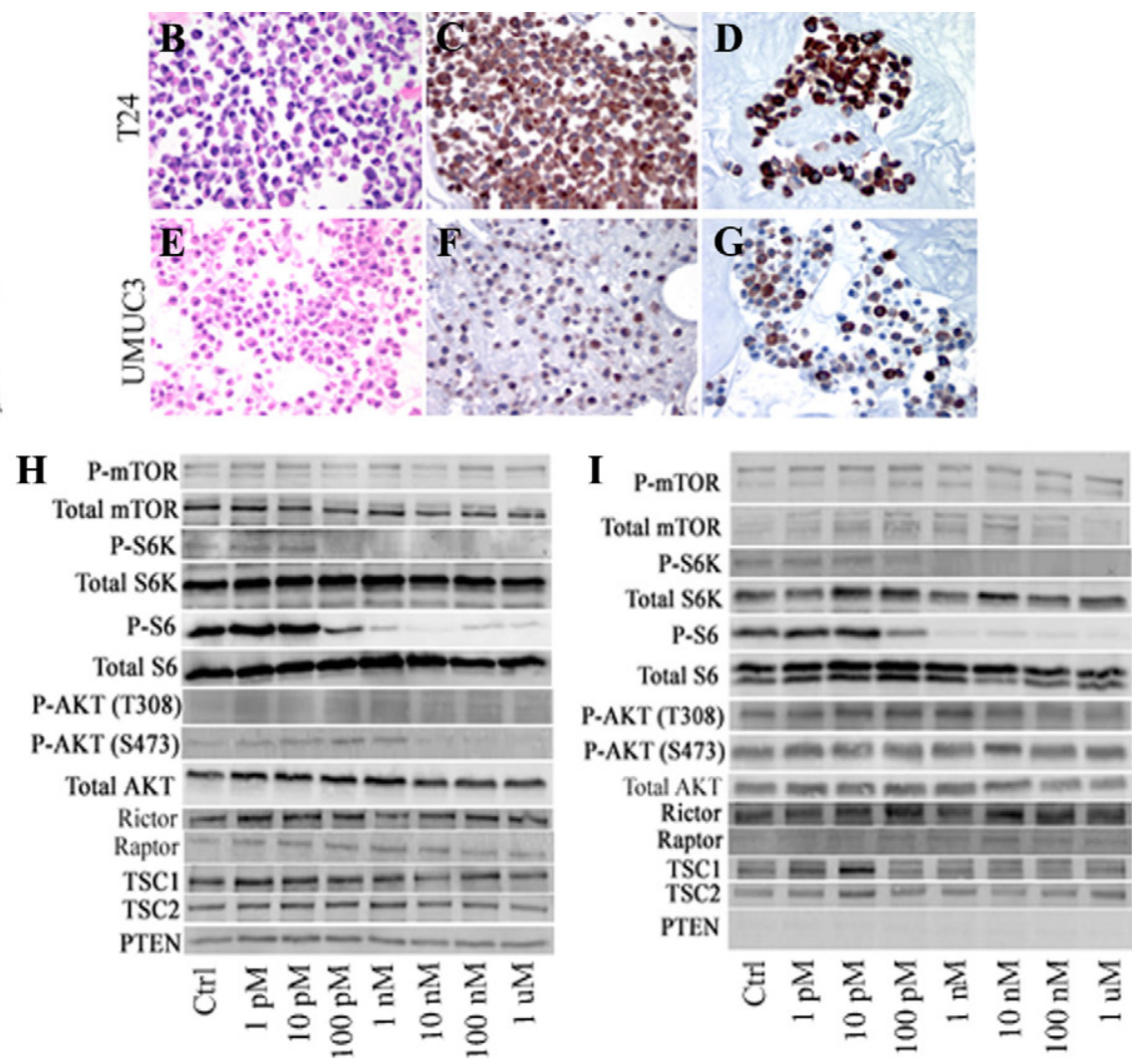

serum-starved for 24 hours and incubated with $1 \mathrm{pM}$ to 1 $\mu \mathrm{mol} / \mathrm{L}$ rapamycin for 48 hours. Control cells were incubated under parallel conditions with the solvent DMSO. Rapamycin treatment resulted in a dose-dependent decrease in proliferation within the nanomolar range of drug $^{20}$ (Figure 5A). Control cells demonstrated a baseline proliferative index of $41.9 \%$ (J82), 58.4\% (T24), $13.1 \%$ (RT4), and $26.2 \%$ (UMUC3) of the total cell population. Addition of $1 \mathrm{nmol} / \mathrm{L}$ rapamycin reduced the proliferation index by 57\% (J82), 94\% (T24), 45\% (RT4), and $29 \%$ (UMUC3) of control, which was statistically significant by Student $t$ test in J82, T24, and RT4 cells $(P<$ $0.01, P<0.01, P=0.03$, and $P=0.14$, respectively). A statistically significant reduction in UMUC3 proliferation with rapamycin treatment was evident at $10 \mathrm{nmol} / \mathrm{L}$ drug $(P=0.03)$.

To determine whether the effects seen were specific for mTOR, we used control and scrambled mTOR siRNA to test effects of rapamycin on cell lines. Treatment of T24 and UMUC3 cells with mTOR-specific siRNAI successfully reduced baseline levels of mTOR by Western blot analysis (see Supplemental Figure S1 at http://ajp. amjpathol.org). Treatment of cells with mTOR siRNA resulted in a reduction of BrdU-labeled cells by $23 \%$ in T24 cells and $53 \%$ in UMUC3 cells relative to control scrambled siRNA, indicating a baseline function for mTOR in mediating aspects of cell proliferation in vitro. However, no further decrease in proliferation between scrambled and mTOR siRNA-treated cells was evident in either T24 or UMUC3 cells treated with $1 \mathrm{pM}, 1 \mathrm{nmol} / \mathrm{L}$, or $1 \mu \mathrm{mol} / \mathrm{L}$ rapamycin, suggesting that the rapamycin effects on
BrdU proliferation were relatively specific for the mTOR signaling pathway. Analysis of cells treated in parallel by TUNEL labeling and PARP cleavage (see Supplemental Figure S2 at http://ajp.amjpathol.org) showed no effect on apoptosis in these assays.

To validate our immunohistochemical technique used on human tissue, we generated paraffin-embedded cell blocks from our cultured cells to examine P-mTOR and P-S6 immunostaining intensity and \% positive cells. As anticipated, the robust reduction in cell proliferation seen with T24 cells was associated with robust and diffuse P-mTOR and P-S6 expression in this cell population (Figure 5, B-D). In contrast, UMUC3 cells, which demonstrate a modest but significant decrease in cell proliferation with rapamycin, show weaker and more restricted expression of both P-mTOR and P-S6 (Figure $5, E-G$ ). These results suggest that intensity and extent of pathway activation, as determined by immunohistochemistry, may indicate potential response to mTOR inhibition.

We next evaluated mTOR pathway components, as well as S6 phosphorylation change with rapamycin treatment, in T24 and UMUC3 cells (Figure 5, H and I). Expression of mTOR, S6K, ribosomal S6 protein, AKT, Rictor, Raptor, TSC1, and TSC2 were present in both cell types, consistent with expression of both mTORC1 and mTORC2 signaling pathways. PTEN gene analysis of T24 cells revealed a single point mutation within exon 2 (c.132 C>T Gly44Gly), a previously reported nonsense mutation. ${ }^{21}$ In contrast, our UMUC3 cell line demonstrated homozygous deletion of the PTEN gene, ${ }^{21,22}$ which has 
A
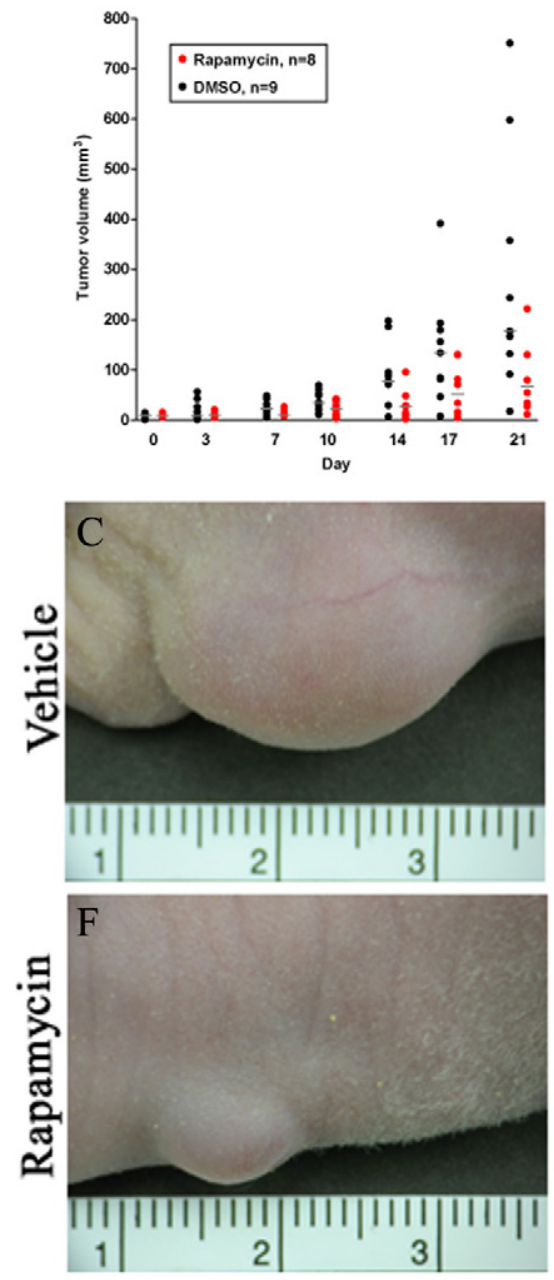

B

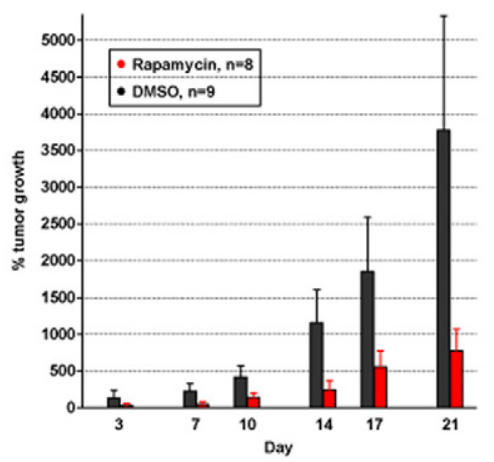

Figure 6. Administration of intraperitoneal rapamycin twice weekly on mice containing T24 xenografts showed a statistically significant reduction in mean tumor volume (A; dots represent individual animals, horizontal bars represent average) and percent tumor growth from initial measurement relative to vehicle-treated animals $(\mathbf{B})$. C and $\mathbf{D}$ : Representative images from vehicle-treated mice are associated with brisk mitotic activity, demonstrated by Ki-67 immunostain (E). In contrast, rapamycin-treated mice had a visible reduction in tumor growth that was associated with a significant reduction in Ki-67 labeling $(\mathbf{F}-\mathbf{H})$.
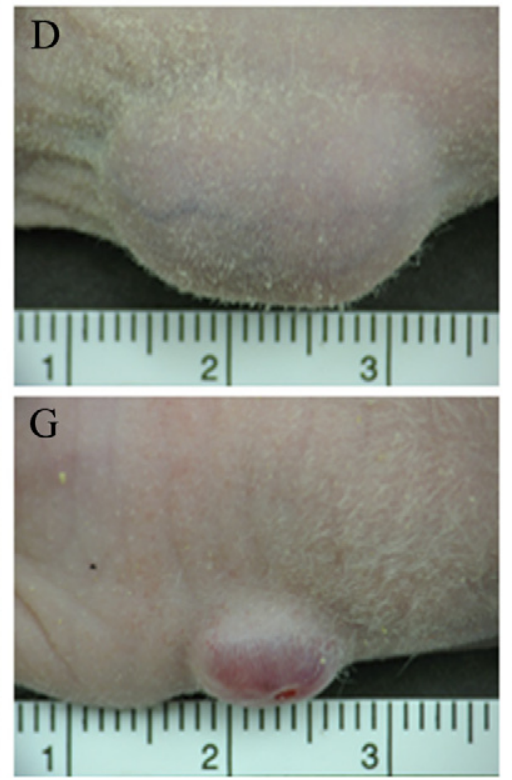
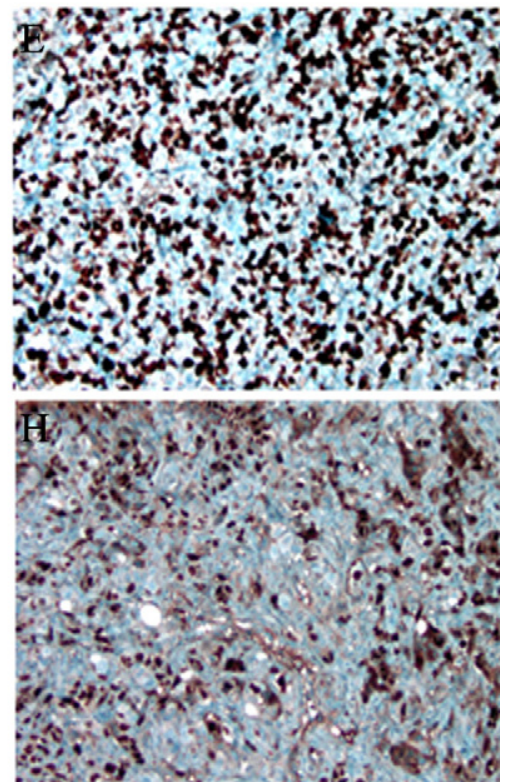

been previously documented and results in complete loss of the PTEN protein product.

Parallel T24 and UMUC3 cell cultures were treated with either DMSO vehicle or increasing doses of rapamycin for 48 hours, and were subsequently collected and phosphorylation cascades analyzed by Western blot analysis. In both cell lines, a dose-dependent decrease in phosphorylation of S6K and S6 after rapamycin was present in both cell lines, with the most striking reduction occurring at the $100 \mathrm{pM}$ to $10 \mathrm{nmol} / \mathrm{L}$ range. Despite loss of PTEN in UMUC3 cells, response to rapamycin administration appeared unaffected. Finally, although rapamycin appeared to mediate mTOR downstream signaling, we also evaluated phosphorylation of AKT on both Ser473 and Thr308 residues, as prolonged rapamycin exposure may variably impact AKT independently in a cell-specific manner. ${ }^{23}$ Results from this study suggest that a mild reduction in Ser473 phosphorylation occurs at higher rapamycin concentrations, although this appears to be at a higher concentration than that required for maximal effects on S6K and S6.
Rapamycin Treatment of T24-Xenografted Mice Reduces Tumor Size

We further evaluated the ability of rapamycin to reduce tumor growth in a mouse xenograft model after subcutaneous inoculation of T24 cells. Once tumors were palpable, mice were administered intraperitoneal rapamycin at $2.5 \mathrm{mg} / \mathrm{kg}(n=8)$ or vehicle control $(n=9)$ twice weekly for 4 weeks. By study completion, mean tumor volume for the control group was $281 \mathrm{~mm}^{3}$ (median tumor volume $177 \mathrm{~mm}^{3}$ ) versus $126 \mathrm{~mm}^{3}$ mean tumor volume in the rapamycin treated group (median tumor volume $80 \mathrm{~mm}^{3}$; $P=0.03$; Figure $6 \mathrm{~A}$ ). Comparison of $\%$ tumor growth between final and initial tumor volumes indicates an average $100 \%$ tumor growth in the rapamycin-treated population and an average $280 \%$ tumor growth in the control group (Figure 6B). Representative images of control (Figure 6, C and D) and rapamycin treated animals (Figure 6, $F$ and $G$ ) show marked differences in visible tumor size. Immunohistochemical analysis for Ki-67, which correlates with nuclear localization during active phases of the cell cycle, ${ }^{24}$ was next performed to evaluate the differences 
in proliferative fractions between vehicle- and rapamycintreated mice. Control animals demonstrated an average of 162 Ki-67-labeled nuclei per high-power field (Figure $6 \mathrm{E})$, whereas proliferation indices were reduced in rapamycin-treated animals to $97 \mathrm{Ki}-67-l a b e l e d$ nuclei per high-power field $(P<0.01$; Figure $6 \mathrm{H})$.

\section{Discussion}

Based on its role as a key downstream effector of AKT, mTOR activity has been examined in a wide array of cancer types, ${ }^{7,8}$ and inhibition of mTOR signaling has been shown to reduce tumor growth. ${ }^{25,26}$ Several recent publications on UCC have identified alterations in upstream molecules involved in mTOR signaling, suggesting that disruption of the mTOR pathway may occur at multiple steps. Approximately $30 \%$ of UCCs have been shown to demonstrate either loss-of-heterozygosity or homozygous deletion of the PTEN locus, and occasional mutations in the PTEN coding region can occur. ${ }^{27} \mathrm{Sev}$ eral recent studies identified that PTEN protein loss appeared to correlate with increased mTOR activity in UCCs arising in the renal pelvis and in a mouse model of UCC, suggesting a direct effect of PTEN on downstream signaling components in this tumor system..$^{28,29}$ In addition, p53 loss-a common occurrence in high-grade UCC-appears to synergize with PTEN loss to promote activation of the mTOR signaling pathway. ${ }^{29}$ Finally, lossof-heterozygosity of TSC1 (9q34), a 23-exon gene that encodes one of the subunits of the TSC1/TSC2 heterodimer, has been reported in approximately $50 \%$ of cases, in addition to numerous missense, nonsense, splicing mutations, and small deletions, ${ }^{30,31}$ although no defined hotspot has been identified. A small subset of these mutations results in reduced TSC 1 protein stability and altered cellular localization, which may ultimately impact mTOR signaling. ${ }^{30}$ Taken together, dysregulation of upstream mTOR pathway components have been reported in UCC and suggest that mTOR signaling may be increased in this cancer type.

For this study, we primarily used high-grade, highstage UCCs for which radical cystectomy is the primary treatment modality and for which new therapies are most needed. The frequent expression of P-mTOR and P-S6 in these specimens suggests that a large proportion of patients may indeed have increased activity of the mTOR signaling cascade, with the potential to respond to mTOR inhibitor therapy, which was the focus of our cell line analysis. In addition, mTOR activity appears to occur as well in many cases of non-invasive low and high grade UCC, suggesting that activation of this pathway may represent a relatively early step in urothelial carcinogenesis. Although the upstream factor(s) that are responsible for the differential activation of this pathway in human UCC is currently unclear at this time, they are likely to include PTEN and TSC1 alterations, as well as differential extracellular signaling cascades.

When analyzed relative to clinicopathologic parameters and patient survival, increased P-mTOR status, either by intensity or percentage of positive cells, appeared to be most significantly associated with increased pathological stage (depth of invasion into the bladder wall) and with shortened patient survival. These findings parallel those of other studies, in which mTOR activation in cancer cells has been associated with more aggressive biological behavior, including in cervical and gastric cancer. ${ }^{32-34}$ These observations are supported by our findings that rapamycin can inhibit UCC migration in vitro, a relevant correlate to depth of invasion in human specimens. Although P-S6 intensity demonstrated a trend toward increased pathological stage, no significant correlation between P-S6 status and other clinicopathologic features or survival time was identified.

The difference between P-mTOR and P-S6 status in a subset of cases suggests that an additional level of complexity may play a role in the ultimate downstream signaling of mTOR, such as regulation of S6 phosphorylation via PDK1, although we have not yet investigated this aspect. Finally, we have used phospho-specific antibodies in our analysis of human tumor specimens, which may prove challenging in immunohistochemical analysis. However, the lack of nonspecific background staining, in comparison with in vitro cell cultures processed in a parallel manner and analyzed by comparative Western blot analysis and rigorous use of biospecimen collection protocols suggests that our data accurately depicts the phosphorylation status of mTOR components in our specimens.

Investigation of the effects of mTOR inhibition via rapamycin, which associates with the cytosolic protein FKBP12 to generate a complex that binds and inhibits mTORC1, was performed on UCC in vitro and in mouse xenograft models. In general, all components of the mTOR signaling pathway were present within the T24 and UMUC3 cells tested, with the exception of PTEN in the latter cell line. Of note, the presence or absence of PTEN status in our cell lines did not appear to affect the ability to inhibit mTOR signaling, although we did not address the effects of PTEN loss on baseline cellular proliferation. All UCC cell lines tested in vitro demonstrated a dosedependent reduction in cell proliferation, which paralleled reduction in P-S6K and P-S6 status by Western blot, suggesting an association between a reduction in cell growth and inhibition of mTOR signaling. Furthermore, immunohistochemical analysis of the phosphorylation status of both P-mTOR and P-S6 appeared to correlate with responsiveness of cell growth to mTOR inhibition. One recent study has examined the effects of mTOR inhibition via rapamycin on UCC cells in vitro and showed reduced cell viability via microculture tetrazolium assay with rapamycin, although the effects on target phosphorylation were not demonstrated and rapamycin concentrations as high as $10 \mu \mathrm{mol} / \mathrm{L}$ and $100 \mu \mathrm{mol} / \mathrm{L}$ were used, ${ }^{35}$ thus possibly resulting in AKT-dependent effects rather than selective mTOR inhibition as well as possibly inducing cell toxicity. In our study, no effects on apoptosis were noted in our rapamycin-treatment group by either TUNEL labeling or PARP cleavage, and it thus appears that reduced cell numbers in vitro are a result of reduced proliferation rather than cell death. 
We identified a reduction in S6K and S6 phosphorylation in our cell lines after rapamycin administration, suggesting that in UCC cells mTORC1 is a primary target of the rapamycin/FKBP12 complex. One recent study, however, suggests that rapamycin may variably impact AKT signaling with prolonged exposure. ${ }^{23}$ We evaluated this reported finding in our analysis and did identify a slight reduction in Ser473 AKT phosphorylation at higher concentrations of rapamycin-concentrations that were higher than those required to fully ablate S6 phosphorylation. Thus, at the lower concentrations of rapamycin (10 $\mathrm{nmol} / \mathrm{L}$ or less) at which we identified reduced proliferation, direct effects on AKT were unlikely.

In support of our in vitro data, treatment of mice bearing subcutaneous T24 xenografts resulted in a 55\% reduction in tumor size over a short time period, suggesting systemic administration of rapamycin may induce tumorspecific effects. These results are supported by the finding that Ki-67 labeling was concurrently reduced by $40 \%$ in rapamycin-treated animals, suggesting that a reduction in tumor size may be at least in part the result of changes in cell proliferation.

Based on the frequent finding of mTOR pathway activation in UCC and the striking reduction in cell proliferation with mTOR inhibition, this pathway may represent a novel therapeutic avenue in the treatment of UCC. This report represents one of the first detailed mTOR pathway analyses in this tumor type. Clinical translation of these findings will require additional analysis of the utility of rapamycin analogs (RAD001, CCl-779) to predict responsiveness to drug, further analysis of upstream signaling components in relation to mTOR signaling in UCC, and evaluation of the tumor microenvironment, including hypoxia, in affecting the mTORC1 pathway. ${ }^{36-38}$

\section{References}

1. Eble JN SG, Epstein JI, Sesterhenn IA: Pathology and genetics of tumours of the urinary system and male genital organs. In: Kleiheus $P$ SL (ed.) World Health Organization Classification of Tumours, Lyon, France: IARC Press 2004

2. Stoter G, Splinter TA, Child JA, Fossa SD, Denis L, van Oosterom AT, de Pauw M, Sylvester R: Combination chemotherapy with cisplatin and methotrexate in advanced transitional cell cancer of the bladder. J Urol 1987, 137:663-667

3. Wu XR: Urothelial tumorigenesis: a tale of divergent pathways. Nat Rev Cancer 2005, 5:713-725

4. Saxman SB, Propert KJ, Einhorn LH, Crawford ED, Tannock I, Raghavan D, Loehrer PJ Sr, Trump D: Long-term follow-up of a phase III intergroup study of cisplatin alone or in combination with methotrexate, vinblastine, and doxorubicin in patients with metastatic urothelial carcinoma: a cooperative group study. J Clin Oncol 1997, 15:25642569

5. Sternberg CN, Donat SM, Bellmunt J, Millikan RE, Stadler W, De Mulder P, Sherif A, von der Maase H, Tsukamoto T, Soloway MS: Chemotherapy for bladder cancer: treatment guidelines for neoadjuvant chemotherapy, bladder preservation, adjuvant chemotherapy, and metastatic cancer. Urology 2007, 69:62-79

6. Gildea JJ, Herlevsen M, Harding MA, Gulding KM, Moskaluk CA, Frierson HF, Theodorescu D: PTEN can inhibit in vitro organotypic and in vivo orthotopic invasion of human bladder cancer cells even in the absence of its lipid phosphatase activity. Oncogene 2004, 23:6788-6797

7. Atkins MB, Hidalgo M, Stadler WM, Logan TF, Dutcher JP, Hudes GR, Park Y, Liou SH, Marshall B, Boni JP, Dukart G, Sherman ML: Ran- domized phase II study of multiple dose levels of CCl-779, a novel mammalian target of rapamycin kinase inhibitor, in patients with advanced refractory renal cell carcinoma. J Clin Oncol 2004, 22 : 909-918

8. Chan S, Scheulen ME, Johnston S, Mross K, Cardoso F, Dittrich C, Eiermann W, Hess D, Morant R, Semiglazov V, Borner M, Salzberg M, Ostapenko V, Illiger HJ, Behringer D, Bardy-Bouxin N, Boni J, Kong S, Cincotta M, Moore L: Phase II study of temsirolimus (CCl-779), a novel inhibitor of mTOR, in heavily pretreated patients with locally advanced or metastatic breast cancer. J Clin Oncol 2005, 23:5314-5322

9. Drakos E, Rassidakis GZ, Medeiros LJ: Mammalian target of rapamycin (mTOR) pathway signalling in lymphomas. Expert Rev Mol Med 2008, 10:e4

10. Hay N: The Akt-mTOR tango and its relevance to cancer. Cancer Cell 2005, 8:179-183

11. Sabatini DM: mTOR and cancer: insights into a complex relationship. Nat Rev Cancer 2006, 6:729-734

12. Hanna SC, Heathcote SA, Kim WY: mTOR pathway in renal cell carcinoma. Expert Rev Anticancer Ther 2008, 8:283-292

13. Hudes G, Carducci M, Tomczak P, Dutcher J, Figlin R, Kapoor A, Staroslawska E, Sosman J, McDermott D, Bodrogi I, Kovacevic Z, Lesovoy V, Schmidt-Wolf IG, Barbarash O, Gokmen E, O'Toole T, Lustgarten S, Moore L, Motzer RJ: Temsirolimus, interferon alfa, or both for advanced renal-cell carcinoma. N Engl J Med 2007 , 356:2271-2281

14. Nocito A, Bubendorf L, Tinner EM, Süess K, Wagner U, Forster T, Kononen J, Fijan A, Bruderer J, Schmid U, Ackermann D, Maurer R, Alund G, Knönagel H, Rist M, Anabitarte M, Hering F, Hardmeier T, Schoenenberger AJ, Flury R, Jäger P, Fehr JL, Schraml P, Moch H, Mihatsch MJ, Gasser T, Sauter G: Microarrays of bladder cancer tissue are highly representative of proliferation index and histological grade. J Pathol 2001, 94:349-357

15. Mutter GL, Lin MC, Fitzgerald JT, Kum JB, Baak JP, Lees JA, Weng LP, Eng C: Altered PTEN expression as a diagnostic marker for the earliest endometrial precancers. J Natl Cancer Inst 2000, 92:924-930

16. Liaw D, Marsh DJ, Li J, Dahia PL, Wang SI, Zheng Z, Bose S, Call KM, Tsou HC, Peacocke M, Eng C, Parsons R: Germline mutations of the PTEN gene in Cowden disease, an inherited breast and thyroid cancer syndrome. Nat Genet 1997, 16:64-67

17. Birle DC, Hedley DW: Signaling interactions of rapamycin combined with erlotinib in cervical carcinoma xenografts. Mol Cancer Ther 2006, 5:2494-2502

18. Altomare DA, Wang HQ, Skele KL, De Rienzo A, Klein-Szanto AJ, Godwin AK, Testa JR: AKT and mTOR phosphorylation is frequently detected in ovarian cancer and can be targeted to disrupt ovarian tumor cell growth. Oncogene 2004, 23:5853-5857

19. Chiang GG, Abraham RT: Phosphorylation of mammalian target of rapamycin (mTOR) at Ser-2448 is mediated by p70S6 kinase. J Biol Chem 2005, 280:25485-25490

20. Shor B, Zhang WG, Toral-Barza L, Lucas J, Abraham RT, Gibbons JJ, Yu K: A new pharmacologic action of CCl-779 involves FKBP12independent inhibition of mTOR kinase activity and profound repression of global protein synthesis. Cancer Res 2008, 68:2934-2943

21. Liu J, Babaian DC, Liebert M, Steck PA, Kagan J: Inactivation of MMAC1 in bladder transitional-cell carcinoma cell lines and specimens. Mol Carcinog 2000, 29:143-150

22. Aveyard JS, Skilleter A, Habuchi T, Knowles MA: Somatic mutation of PTEN in bladder carcinoma. Br J Cancer 1999, 80:904-908

23. Sarbassov DD, Ali SM, Sengupta S, Sheen JH, Hsu PP, Bagley AF Markhard AL, Sabatini DM: Prolonged rapamycin treatment inhibits mTORC2 assembly and Akt/PKB. Mol Cell 2006, 22:159-168

24. Scholzen T, Gerdes J: The Ki-67 protein: from the known and the unknown. J Cell Physiol 2000, 182:311-322

25. Granville CA, Memmott RM, Gills JJ, Dennis PA: Handicapping the race to develop inhibitors of the phosphoinositide 3-kinase/Akt/mammalian target of rapamycin pathway. Clin Cancer Res 2006, 12: 679-689

26. Podsypanina K, Lee RT, Politis C, Hennessy I, Crane A, Puc J, Neshat M, Wang $H$, Yang L, Gibbons J, Frost P, Dreisbach V, Blenis J, Gaciong Z, Fisher P, Sawyers C, Hedrick-Ellenson L, Parsons R: An inhibitor of mTOR reduces neoplasia and normalizes p70/S6 kinase activity in Pten+/- mice. Proc Natl Acad Sci USA 2001, 98: 10320-10325

27. Cappellen D, Gil Diez de Medina S, Chopin D, Thiery JP, Radvanyi F: 
Frequent loss of heterozygosity on chromosome 10q in muscle-invasive transitional cell carcinomas of the bladder. Oncogene 1997, 14:3059-3066

28. Qian CN, Furge KA, Knol J, Huang D, Chen J, Dykema KJ, Kort EJ, Massie A, Khoo SK, Vanden Beldt K, Resau JH, Anema J, Kahnoski RJ, Morreau H, Camparo P, Comperat E, Sibony M, Denoux Y, Molinie V, Vieillefond A, Eng C, Williams BO, Teh BT: Activation of the PI3K/ AKT pathway induces urothelial carcinoma of the renal pelvis: identification in human tumors and confirmation in animal models. Cancer Res 2009, 69:8256-8264

29. Puzio-Kuter AM, Castillo-Martin M, Kinkade CW, Wang X, Shen TH, Matos T, Shen MM, Cordon-Cardo C, Abate-Shen C: Inactivation of p53 and Pten promotes invasive bladder cancer. Genes Dev 2009, 23:675-680

30. Pymar LS, Platt FM, Askham JM, Morrison EE, Knowles MA: Bladder tumour-derived somatic TSC1 missense mutations cause loss of function via distinct mechanisms. Hum Mol Genet 2008, 17:20062017

31. Cairns P, Shaw ME, Knowles MA: Initiation of bladder cancer may involve deletion of a tumour-suppressor gene on chromosome 9 . Oncogene 1993, 8:1083-1085

32. Hashimoto I, Koizumi K, Tatematsu M, Minami T, Cho S, Takeno N Nakashima A, Sakurai H, Saito S, Tsukada K, Saiki I: Blocking on the
CXCR4/mTOR signalling pathway induces the anti-metastatic properties and autophagic cell death in peritoneal disseminated gastric cancer cells. Eur J Cancer 2008, 44:1022-1029

33. Lamouille S, Derynck R: Cell size and invasion in TGF-beta-induced epithelial to mesenchymal transition is regulated by activation of the mTOR pathway. J Cell Biol 2007, 178:437-451

34. Faried LS, Faried A, Kanuma T, Sano T, Nakazato T, Tamura T, Kuwano $\mathrm{H}$, Minegishi T: Predictive and prognostic role of activated mammalian target of rapamycin in cervical cancer treated with cisplatin-based neoadjuvant chemotherapy. Oncol Rep 2006, 16:57-63

35. Fechner G, Classen K, Schmidt D, Hauser S, Müller SC: Rapamycin inhibits in vitro growth and release of angiogenic factors in human bladder cancer. J Urol 2009, 73:665-668

36. Wouters BG, Koritzinsky M: Hypoxia signalling through mTOR and the unfolded protein response in cancer. Nat Rev Cancer 2008, 8:851-864

37. Arsham AM, Howell JJ, Simon MC: A novel hypoxia-inducible factorindependent hypoxic response regulating mammalian target of rapamycin and its targets. J Biol Chem 2003, 278:29655-29660

38. Li Y, Wang Y, Kim E, Beemiller P, Wang CY, Swanson J, You M, Guan $\mathrm{KL}$ : Bnip3 mediates the hypoxia-induced inhibition on mammalian target of rapamycin by interacting with Rheb. J Biol Chem 2007, 282:35803-35813 